AperTO - Archivio Istituzionale Open Access dell'Università di Torino

\title{
Modelling herd behavior in population systems
}

\section{This is the author's manuscript}

Original Citation:

Availability:

This version is available http://hdl.handle.net/2318/88357

since

Published version:

DOI:10.1016/j.nonrwa.2011.02.002

Terms of use:

Open Access

Anyone can freely access the full text of works made available as "Open Access". Works made available under a Creative Commons license can be used according to the terms and conditions of said license. Use of all other works requires consent of the right holder (author or publisher) if not exempted from copyright protection by the applicable law. 


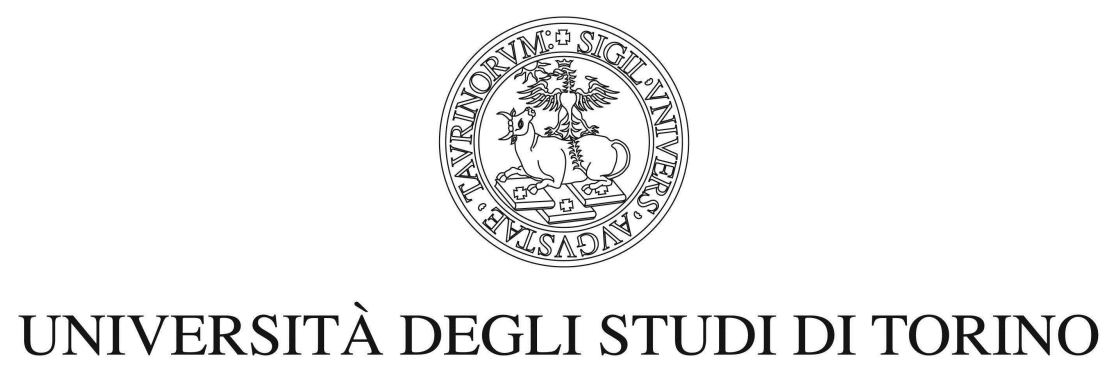

This is an author version of the contribution published on:

Valerio Ajraldi, Marta Pittavino, Ezio Venturino. Modeling herd behavior in population systems. Nonlinear Analysis Real World Applications, 12 (2011) 2319-2338, DOI: 10.1016/j.nonrwa.2011.02.002.

The definitive version is available at:

http://www.journals.elsevier.com/nonlinear-analysis-real-worldapplications/ 


\title{
Modeling herd behavior in population systems
}

\author{
Valerio Ajraldi, Marta Pittavino, Ezio Venturino, \\ Dipartimento di Matematica "Giuseppe Peano", \\ Università di Torino, Via Carlo Alberto 10, \\ 10123 Torino, Italy
}

\begin{abstract}
In this paper we show that under suitable simple assumptions the classical two populations system may exhibit unexpected behaviors. Considering a more elaborated social model, in which the individuals of one population gather together in herds, while the other one shows a more individualistic behavior, we model the fact that interactions among the two occur mainly through the perimeter of the herd. We account for all types of populations interactions, symbiosis, competition and the predator-prey interactions. There is a situation in which competitive exclusion does not hold: the socialized herd behavior prevents the competing individualistic population from becoming extinct. For the predator-prey case, sustained limit cycles are possible, the existence of Hopf bifurcations representing a distinctive feature of this model compared with other classical predator-prey models. The system's behavior is fully captured by just one suitably introduced new threshold parameter, defined in terms of the original model parameters.
\end{abstract}

Keywords: predator-prey, limit cycles, bifurcations, group defense MSC codes: AMS 92D25, 92D50

\section{Introduction}

Two-dimensional dynamical systems modeling populations' interactions are nowadays considered to be classical.

${ }^{*}$ Corresponding author. E-mail: ezio.venturino@unito.it 
The Lotka-Volterra model $[1,2,3,4]$, which is the source of all the research work of the past century in

population models has been modified first to account for possible logistic growth in the prey, so as to avoid the occurrence of neutrally stable oscillations. Then several other possible dynamics have been considered, which depart from the classical assumption of quadratic interactions: we can recall here for instance Holling type II and III functional responses [3, 4, 5], Holling-Tanner systems, $[6,7,8]$ and ratio-dependent models $[9,10]$. The latter, in particular, is subject to discussion among researchers, [11, 12, 13]. Recent and more dated research has in fact evolved toward the modeling of more complex situations, with the aim of better understanding reality, perhaps with the unmentioned assumption that everything is already known on two-dimensional systems. However, quadratic interactions based on the mass-action law are still sometimes used in more complicated models, accounting for food chains [14].

In this paper we take a different, novel view of the classical two population system, showing that under suitable assumptions in some instances it may lead to unexpected behavior. Namely, we model the interactions not just of individuals of two populations that intermingle on a common ground, but consider a more elaborated social model, in which the individuals of one population gather together in herds, to wander about in search of food sources and for defensive purposes. The concept of group defense has already been considered, [15], via suitable assumptions on the form and type of functional responses of the prey, modeled in very general terms. Specifically, there is a threshold on the size of herd of the prey beyond which the predators' hunting capabilities begin to fall. In other words, the larger the prey population is, the smaller the success of hunting and the corresponding return rate are for predators. Here instead we derive the model by observing the behavior of the population which aggregates in herds, having in mind mainly the situation of the herbivores populating the savannas and their large predators. A similar reasoning led in [16] to the formulation of a plankton model in which toxic phytoplankton releases poison through the surface of a threedimensional patch. We look at how the other species deals with it and at the kind of interactions that are possible in such a situation. A different way of interpreting the system consists in observing that our viewpoint tries to describe interactions occurring in space, via a suitable dynamical system in which space does not appear as an independent variable. Rather, it is embedded in the form of the system itself. Mathematically speaking this means to replace a model constructed via partial differential equations by a simpler system of ordinary differential equations, without losing the important features that the actual situation being modeled shows. The basic 
idea is that while one population is assumed to be composed of individuals that essentially live independently of the other ones, the other population instead gathers in herds. The latter for defense purposes usually allows the weakest individuals to occupy the interior of the herd, leaving the healthier and stronger animals around it. What is important in any case, is that the individualistic population interacts

with the more socialized one only through the perimeter of the herd. In particular, in competing models or predator-prey models, when attacks arise, it is mostly the individuals at the border of the herd that suffer the consequences of the predators'

actions. Our aim is to assess how the social behavior ultimately affects the populations interplay. We want to look at all different types of interactions, generalizing the analysis of [17] to all possible situations.

We start from the mutualistic one, leading to symbiotic communities. We then examine the case in which the two populations compete for resources; for recent results on such type of systems see [18]. Finally the predator-prey type of interactions is investigated.

The outcome of the analysis shows that novel features arise, impossible in simple quadratic systems, at least in the last two above scenarios. Certainly some of these results can also be obtained via other types of nonlinearities, but we stress the fact that it is the assumptions we make here that render the system interesting, showing that oscillations observed in the field among interacting populations may be due to factors other than those assumed usually with Holling type II functional responses, i.e. corresponding to the satiation effect a predator experiences when the prey is too much abundant. Furthermore, our investigations indicate that the system's behavior is completely captured by just one suitably introduced new threshold parameter, defined in terms of the original model coefficients.

The paper is organized as follows. In the next Section we briefly review the classical models, for comparison purposes. In Section 3 we discuss the model for symbiotic communities. Section 4 contains the competition system and in the following Section we analyze the predator-prey interactions. A final discussion concludes the paper.

In all the new models we denote by $R$ the highly socialized population and by $F$ the more individualistic one. All the parameters in the models are assumed to be nonnegative. 


\section{Brief review of the classical models}

For later comparison, we present here a quick overview of the less common classical models, [3, 19], avoiding to discuss the well-known Lotka-Volterra predator-prey model, see [20].

\subsection{The classical symbiotic case}

We consider here two populations which both benefit from the mutual interactions. The model is known, therefore we just summarize its formulation and main features.

$$
\begin{aligned}
& \frac{d}{d t} R(t)=r\left(1-\frac{R(t)}{K_{R}}\right) R(t)+a R(t) F(t), \\
& \frac{d}{d t} F(t)=m\left(1-\frac{F(t)}{K_{F}}\right) F(t)+a e R(t) F(t) .
\end{aligned}
$$

In the absence of the other one, each population reproduces logistically, with respective carrying capacities $K_{R}, K_{F}$ and reproduction rates $r, m$, but each gains from the interaction with the other. This fact is expressed by the last terms in the equations. Here the parameter $e$ represents a fraction, which may be larger than one, measuring the relative benefits that the two populations get from their mutual interactions.

The equilibria are the boundary points here reported with their respective eigenvalues $P_{1}^{s}=(0,0), \lambda_{1}=r$ and $\lambda_{2}=m ; P_{2}^{s}=\left(K_{R}, 0\right), \lambda_{1}=-r$ and

$\lambda_{2}=m+a e K_{R} ; P_{3}^{s}=\left(0, K_{F}\right), \lambda_{1}=r+a K_{F}$ and $\lambda_{2}=-m$. The coexistence equilibrium

$$
P_{4}^{s}=\left(\frac{K_{R} m\left(r+a K_{F}\right)}{r m-a^{2} e K_{F} K_{R}}, \frac{K_{F} r\left(m+a e K_{R}\right)}{r m-a^{2} e K_{F} K_{R}}\right) \text {, }
$$

is feasible for $r m \geq a^{2} e K_{F} K_{R}$ and has characteristic equation

$$
\left(r m-a^{2} e K_{F} K_{R}\right) \lambda^{2}+r m\left(m+r+a K_{F}+a e K_{R}\right) \lambda+m r\left(r+a K_{F}\right)\left(m+a e K_{R}\right)=0 .
$$

From the Routh-Hurwitz conditions both eigenvalues have negative real part.

The first three points turn out to be always unstable, while the latter when feasible is inconditionally stable. Note that the system then settles to population levels that are higher than those expressed by their respective carrying capacities, obtained in the absence of the other population. In case $P_{4}^{s}$ results unfeasible, i.e. for $r m<a^{2} e K_{F} K_{R}$, the trajectories are unbounded, an unlikely biological result. 


\subsection{The classical competition model}

We consider now the competition model, in which both populations are harmed by their mutual interactions. A discussion of this model with plots of the phase plane can be found for instance in [19], pages 14-18. Using the same notation as for (1), we have

$$
\begin{aligned}
& \frac{d}{d t} R(t)=r\left(1-\frac{R(t)}{K_{R}}\right) R(t)-a R(t) F(t), \\
& \frac{d}{d t} F(t)=m\left(1-\frac{F(t)}{K_{F}}\right) F(t)-a e R(t) F(t),
\end{aligned}
$$

with boundary equilibria $P_{1}^{c}=(0,0)$, with eigenvalues $\lambda_{1}=r, \lambda_{2}=m$; $P_{2}^{c}=\left(K_{R}, 0\right)$, with eigenvalues $\lambda_{1}=-r, \lambda_{2}=m-a e K_{R} ; P_{3}^{c}=\left(0, K_{F}\right)$ with eigenvalues $\lambda_{1}=r-a K_{F}, \lambda_{2}=-m$; and interior equilibrium

$$
P_{4}^{c}=\left(\frac{r K_{F}\left(a e K_{R}-m\right)}{a^{2} e K_{F} K_{R}-r m}, \frac{m K_{R}\left(a K_{F}-r\right)}{a^{2} e K_{F} K_{R}-r m}\right) .
$$

The latter is feasible if and only if

$$
\operatorname{sign}\left(a e K_{R}-m\right)=\operatorname{sign}\left(a K_{F}-r\right)=\operatorname{sign}\left(a^{2} e K_{F} K_{R}-r m\right) .
$$

Hence, $P_{1}^{c}$ is unstable and the remaining equilibria are all conditionally stable. Namely

$P_{2}^{c}$ is stable if $m<a d K_{R}$,

$P_{3}^{c}$ is stable for $r<a K_{F}$

and $P_{4}^{c}$ requires $d a^{2} K_{F} K_{R}<r m$. When $P_{4}^{c}$ is unstable the principle of competitive exclusion holds, i.e. only one of the two populations can survive in this context, depending on the initial conditions of the system. Furthermore, the Dulac's criterion prevents limit cycles, [19], p. 15. A recent result for more complex situation can be found in [21].

\section{The new symbiotic model}

Here we introduce the herd behavior, as follows. If we consider $R(\tau)$ to represent the density of the first population, namely number of individuals per surface unit, with the herd occupying an area $A$, it follows that the individuals who take the outermost positions in the herd are proportional to the perimeter of the patch where the herd is located whose length depends on $\sqrt{A}$. They are therefore in number proportional to the square root of the density, i.e. to $\sqrt{R}$, with a proportionality constant depending on the 
shape of the herd. In practice this could be regarded as a particular form of the Gompertz law, with the special exponent $\gamma=\frac{1}{2}$. The interactions with the second populations occur only via these periferical individuals, so that instead of the standard mass-action term giving the quadratic type of interaction of (1), we find a term proportional to $\sqrt{R} F$. The model then reads

$$
\begin{aligned}
& \frac{d}{d \tau} R(\tau)=r\left(1-\frac{R(\tau)}{K_{R}}\right) R(\tau)+\widetilde{a} \sqrt{R(\tau)} F(\tau), \\
& \frac{d}{d \tau} F(\tau)=\widetilde{m}\left(1-\frac{F(\tau)}{K_{F}}\right) F(\tau)+\widetilde{a} \widetilde{e} \sqrt{R(\tau)} F(\tau),
\end{aligned}
$$

where the first equation describes the evolution of the highly socialized population. It reproduces logistically and, as mentioned, it interacts with the second one only through the individuals lying at the outskirts of the herd. The second population grows also logistically and benefits from the interactions via a factor $\widetilde{e}>0$.

\subsection{Model simplification}

The model (3) is easily seen to possess a singularity in the Jacobian, due to the square root term. It is therefore advisable to remove it, before proceeding to the analysis. Let us define the new dependent variable $P(\tau)=\sqrt{R(\tau)}$. After simplification, (3) becomes

$$
\begin{aligned}
& \frac{d}{d \tau} P(\tau)=\frac{r}{2}\left(1-\frac{P(\tau)^{2}}{K_{R}}\right) P(\tau)+\frac{\widetilde{a}}{2} F(\tau), \\
& \frac{d}{d \tau} F(\tau)=\widetilde{m}\left(1-\frac{F(\tau)}{K_{F}}\right) F(\tau)+\widetilde{a} \widetilde{e} P(\tau) F(\tau) .
\end{aligned}
$$

Note that here we have divided the first equation by $P$. However, if $P \equiv$ 0 , the first equation becomes an identity, and the system reduces just to the evolution equation for $F$. This is to be taken into account in the next Subsection, when analyzing the equilibria.

If we rescale the variables as follows

$$
p=\frac{P}{K_{P}}, \quad f=\frac{F}{K_{F}}, \quad t=\frac{r \tau}{2},
$$

and define the new parameters

$$
a=\frac{\widetilde{a} K_{F}}{r \sqrt{K_{R}}}, \quad m=\frac{2 \widetilde{m}}{r}, \quad e=\frac{2 \widetilde{a} \widetilde{e} \sqrt{K_{R}}}{r},
$$


the adimensionalized system can be written as

$$
\begin{aligned}
& \frac{d}{d t} p(t)=\left(1-p(t)^{2}\right) p(t)+a f(t), \\
& \frac{d}{d t} f(t)=m(1-f(t)) f(t)+e p(t) f(t) .
\end{aligned}
$$

\subsection{Equilibria}

The equilibria are the boundary points $P_{1}^{S}=(0,0), P_{2}^{S}=(1,0), P_{3}^{S}=(0,1)$, with the last one arising from the above mentioned fact that for $p \equiv 0$ the first equation is an identity. The latter two equilibria correspond to the points $\left(K_{R}, 0\right)$ and $\left(0, K_{F}\right)$ in the original model. The coexistence equilibrium $P_{4}^{S}=\left(p_{P}, f_{P}\right)$ arises from the roots of the cubic

$$
\Psi(p) \equiv-p^{3}+\left(1+\frac{a e}{m}\right) p+a .
$$

Since $\Psi(0)>0$ and $\Psi^{\prime}(0)>0$ there is only one positive root, leading to only one coexistence equilibrium, with

$$
f_{P}=\frac{1}{a} p_{P}\left(p_{P}^{2}-1\right)=1+\frac{e}{m} p_{P} \geq 0
$$

showing that it is always feasible. But from the first equation (6) for the prey population we obtain the lower bound

$$
p_{P} \geq 1
$$

\subsection{Stability}

The Jacobian of (3) is

$$
\left(\begin{array}{cc}
1-3 p^{2} & a \\
e f & m(1-2 f)+e p
\end{array}\right)
$$

At the origin $P_{1}^{S}$, we find the eigenvalues $\lambda_{1}=1$ and $\lambda_{2}=m$, showing its instability.

At $P_{2}^{S}$ the characteristic equation factors, giving the eigenvalues $\lambda_{1}=-2$ and $\lambda_{2}=m+e>0$ from which instability follows.

At $P_{3}^{S}$ the system degenerates into only the second equation (5) for which on the line $p=0$ the point $P_{3}^{S}$ is a stable equilibrium. However, since at any point $\left(\epsilon, f^{0}\right)$, with $\epsilon>0$ but arbitrarily small and $f^{0}$ arbitrary we have 
$\frac{d}{d t} p=a f^{0}+O(\epsilon)>0$, it follows that $P_{3}^{S}$ cannot be stable in the $p-f$ phase plane.

The explicit coordinates of $P_{4}^{S}$ are not known, but from the phase plane analysis, see Figure $1, P_{4}^{S}$ is seen to be a stable node. This conclusion is also algebraically confirmed, from the Routh-Hurwitz criterion applied to the characteristic equation, giving

$$
\begin{array}{r}
(\mathrm{a}):-\operatorname{tr}\left(J\left(P_{4}^{S}\right)\right) \equiv \phi\left(p_{P}\right)=3 p_{P}^{2}-1+m f_{P}>0, \\
(\mathrm{~b}): \operatorname{det}\left(J\left(P_{4}^{S}\right)\right) \equiv \Phi\left(p_{P}\right)=-m f_{P}\left[1-3 p_{P}^{2}\right]-a e f_{P}>0 .
\end{array}
$$

The first one, which using (7) can be explicitly rewritten as

$$
\phi\left(p_{P}\right)=3 p_{P}^{2}-1+m f_{P}>2+m f_{P}>0,
$$

is then found to hold always. Condition (a) holds then unconditionally.

For (b), using both equations (6) in its definition and the bound (7), the cubic $\Phi$ explicitly becomes

$\Phi\left(p_{P}\right)=\left(e p_{P}+m\right)\left[3 p_{P}^{2}-1\right]+e p_{P}\left(1-p_{P}^{2}\right)=p_{P}^{2}\left(2 e p_{P}+3 m\right)-m>2 e p_{P}^{3}+2 m>0$.

Hence also the second Routh-Hurwitz condition holds inconditionally.

We now show an important result for this model.

Take a point $\widehat{P}=(\widehat{p}, \widehat{f})$ in the phase plane, with $\widehat{p}>p_{P}, \widehat{f}>f_{P}$ and lying below the isocline $\frac{d p}{d t}=0$ and above the isocline $\frac{d f}{d t}=0$, thus for which the inequalities

$$
\left(1-\widehat{p}^{2}\right) \widehat{p}+a \widehat{f}<0, \quad m(1-\widehat{f})+e \widehat{p}<0
$$

hold. It identifies a rectangle $\Omega$ in the phase plane, with opposite vertex given by the origin. This set $\Omega$ is a positively invariant set for the dynamical system (5). In fact the coordinate axes cannot be crossed by the existence and uniqueness theorem, since the system is homogeneous and therefore they are solution trajectories. Furthermore on the vertical line $p=\widehat{p}$ we have $\frac{d p}{d t}<0$ while instead $\frac{d f}{d t}<0$ on the horizontal line $f=\widehat{f}$. Hence the flow of (5) enters into $\Omega$ from these sides. Furthermore all system's trajectories must enter it, since the chosen point $\widehat{P}$ lying on the north-east of $P_{4}^{S}$ is arbitrary. Thus letting $\left(p^{(0)}, f^{(0)}\right)$ denote the initial point for any trajectory, by choosing $\widehat{p}>^{(0)}, \widehat{f}>f^{(0)}$ we can ensure that the trajectory lies entirely in $\Omega$.

Introducing now the function $B(p, f)=(p f)^{-1}$ and calculating the ex- 
pression

$$
\begin{array}{r}
\frac{\partial}{\partial p}\left[B(p, f) \frac{d p}{d t}\right]+\frac{\partial}{\partial f}\left[B(p, f) \frac{d f}{d t}\right] \\
=\frac{\partial}{\partial p}\left[\frac{1}{p f}\left(p\left(1-p^{2}\right)+a f\right)\right]+\frac{\partial}{\partial f}\left[\frac{1}{p f}(m f(1-f)+e p f)\right] \\
=-2 \frac{p}{f}-\frac{a}{p^{2}}-\frac{m}{p}<0
\end{array}
$$

we discover that it is negative in $\Omega$, therefore by Dulac's theorem, no periodic orbit of (5) can exist in $\Omega$. Since the equilibrium $P_{4}^{S}$ is locally asymptotically stable, all the other ones are unstable, and no periodic orbit can exist in $\Omega$, it follows that $P_{4}^{S}$ must also be globally asymptotically stable.

In summary we have the following theorem.

Theorem 1. The coexistence equilibrium $P_{4}^{S}$ of the system (5) is globally asymptotically stable.

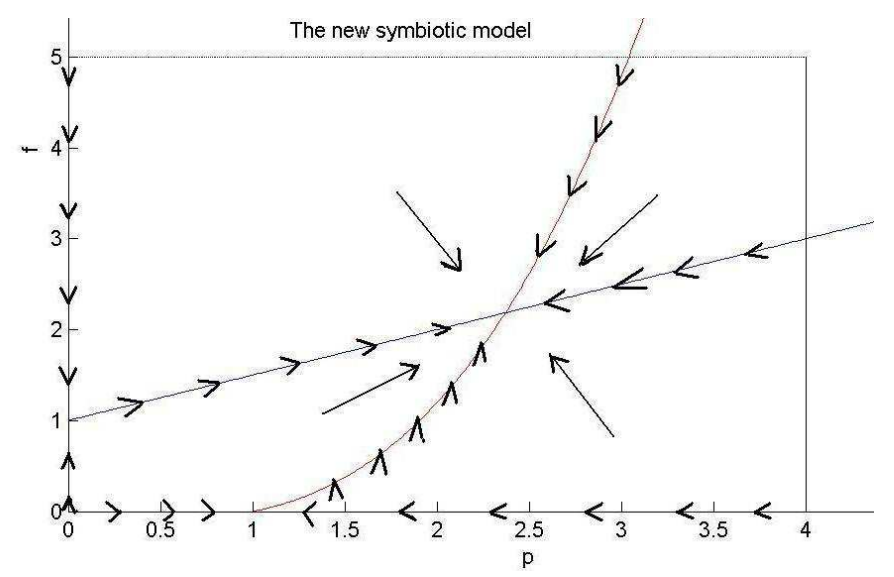

Figure 1: Symbiotic model (5). Parameter values: $a=5, m=6, e=3$.

\subsection{Obligated mutualism for the solitary population}

A variation of the model (3) can be introduced by considering obligated mutualism. We assume it only for the second population; no relevant changes occur when it is assumed for both populations. The model gets modified as follows

$$
\begin{aligned}
& \frac{d}{d \tau} R(\tau)=r\left(1-\frac{R(\tau)}{K}\right) R(\tau)+a \sqrt{R(\tau)} F(\tau), \\
& \frac{d}{d \tau} F(\tau)=-\widetilde{m} F(\tau)+a \widetilde{e} \sqrt{R(\tau)} F(\tau) .
\end{aligned}
$$




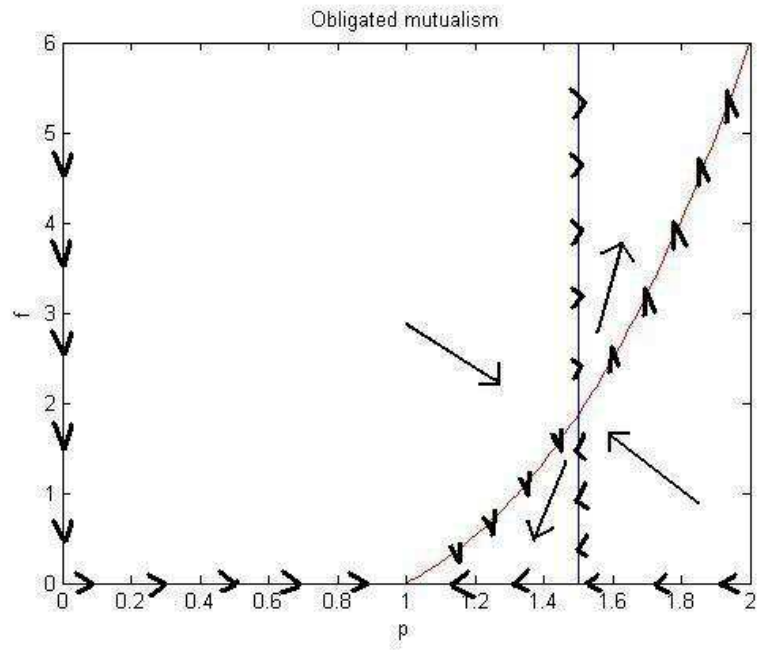

Figure 2: Phase space diagram of the obligated mutualistic system (10) for the parameter values $m=1.5, e=1$ implying $\rho=1.5$.

The singularity can be removed and the system can be adimensionalized in a similar way as for (3), with

$$
m=\frac{2 \widetilde{m}}{r}, \quad e=\frac{2 a \widetilde{e} \sqrt{K}}{r},
$$

to get

$$
\begin{aligned}
& \frac{d}{d t} p(t)=p\left(1-p^{2}\right)+f \\
& \frac{d}{d t} f(t)=(e p-m) f
\end{aligned}
$$

with Jacobian

$$
\widetilde{J} \equiv\left(\begin{array}{cc}
1-3 p^{2} & 1 \\
e f & e p-m
\end{array}\right) .
$$

Let us define the parameter

$$
\rho=\frac{m}{e}
$$

The boundary equilibria are the origin, always unstable, and the point $\widetilde{P}_{2} \equiv$ $(1,0)$, stable if $\rho>1$. Note that in this case the point $P_{3}^{S}=(0,1)$ is not an equilibrium, contrary to what happens when symbiosis is not obligated. This is clearly biologically reasonable, since $P_{3}^{S}$ corresponds to the extinction of 


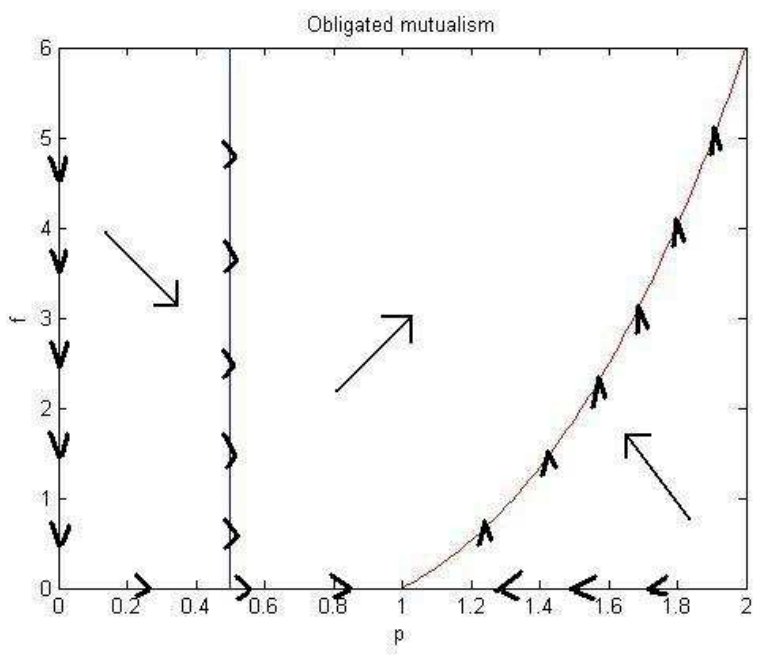

Figure 3: Phase plane diagram of the obligated mutualistic system (10) for the parameter values $m=0.5, e=1$ implying $\rho=0.5$.

the population $p$ that is necessary for the survival of $f$, the species for which mutualism is obligated and therefore the latter cannot survive. Further, there is the coexistence equilibrium $\widetilde{P}_{4} \equiv(\widetilde{p}, \widetilde{f}) \equiv\left(\rho, \rho\left(\rho^{2}-1\right)\right)$, feasible for $\rho>1$. It turns out to have always a positive eigenvalue though, since the determinant of the Jacobian is negative, $\widetilde{J}\left(\widetilde{P}_{4}\right)=-e \widetilde{f}<0$. Thus the coexistence equilibrium is always unstable, Figure 2.

In summary, for $\rho>1$, equilibrium $\widetilde{P}_{2}$ is feasible and locally asymptotically stable, while $\widetilde{P}_{4}$ is feasible and unstable; more specifically it is a saddle and above the separatrix the system's trajectories tend to infinity. For $\rho<1$ instead no stable equilibria exist since $\widetilde{P}_{2}$ becomes unstable and $\widetilde{P}_{4}$ infeasible; all the trajectories of the system (10) then tend to infinity,

This is an unlikely situation from the biological point of view, but mathematically it can occur, as shown in Figure 3.

\section{The competition model}

We consider here two populations fighting for the same resources. Again $R$ denotes the highly socialized one, living and wandering in herds. The $F$ population is instead once again the more lonely one and the interactions among the two occur only at the boundary of the herd, therefore involving only the individuals of $R$ who generally occupy positions at the margin of 
the herd. The model reads

$$
\begin{aligned}
& \frac{d}{d t} R(t)=r\left(1-\frac{R(t)}{K_{R}}\right) R(t)-\widetilde{a} \sqrt{R(t)} F(t), \\
& \frac{d}{d t} F(t)=\widetilde{m}\left(1-\frac{F(t)}{K_{F}}\right) F(t)-\widetilde{a} \widetilde{e} \sqrt{R(t)} F(t) .
\end{aligned}
$$

Upon rescaling, proceeding as for the model (3), we find the following adimensionalized model

$$
\begin{aligned}
& \frac{d}{d t} p(t)=p\left(1-p^{2}\right)-a f \\
& \frac{d}{d t} f(t)=m f(1-f)-e p f .
\end{aligned}
$$

\subsection{Equilibria}

We find the boundary points $P_{1}^{C}=(0,0), P_{2}^{C}=(1,0), P_{3}^{C}=(0,1)$, the latter arising as a special case, since for $p \equiv 0$ the first equation becomes an identity. The coexistence equilibrium is obtained from the roots of the following cubic

$$
\Pi(p)=p^{3}-\left(1+\frac{a e}{m}\right) p+a=0 .
$$

By Descartes' rule, there are either two positive roots, $\widetilde{p}_{ \pm}$, or none. Hence possibly two equilibria, $P_{4 \pm}$. Precisely, since $\Pi(0)>0$ and $\Pi^{\prime}(0)<0$, letting

$$
\bar{p}=\sqrt{\frac{1}{3}\left(1+\frac{a e}{m}\right)}
$$

this value being defined by $\Pi^{\prime}(\bar{p})=0$, there are two roots if and only if $\Pi(\bar{p})<0$ which amounts to

$$
\bar{p}^{3} \geq \frac{1}{2} a
$$

The latter is the existence condition for the interior equilibria $P_{4 \pm}=\left(\widetilde{p}_{ \pm}, \widetilde{f}_{ \pm}\right)$, with predators' level given by

$$
f_{ \pm}=\frac{1}{a}\left(1-\widetilde{p}_{ \pm}^{2}\right) \widetilde{p}_{ \pm}=1-\frac{e}{m} \widetilde{p}_{ \pm}
$$

From this, feasibility requires then that $\widetilde{p}_{ \pm} \leq 1, \widetilde{p}_{ \pm} \leq \rho$ so that combining the two, we get

$$
\widetilde{p}_{ \pm} \leq \min \{1, \rho\}
$$

Also, note that $\widetilde{p}_{-}<\bar{p}<\widetilde{p}_{+}$. 


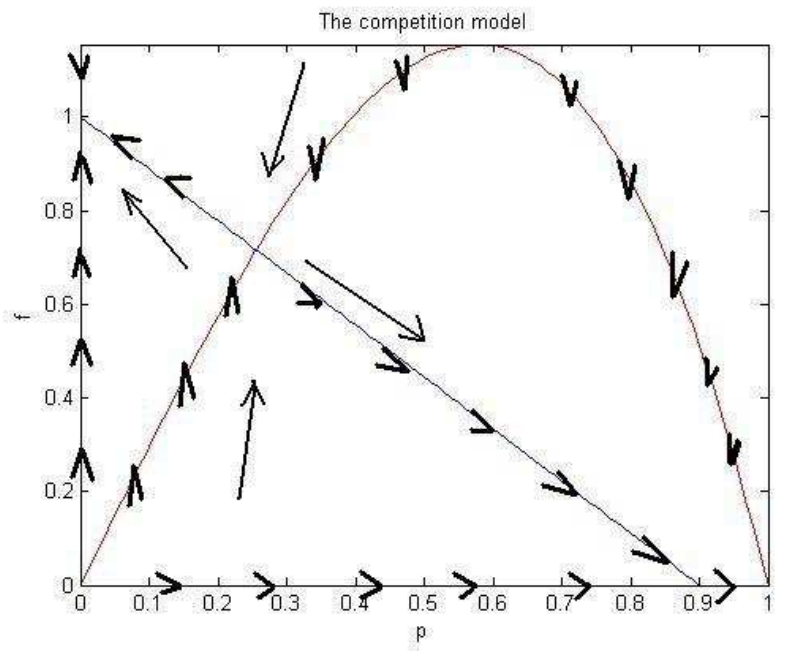

Figure 4: Competition model (14). Phase plane diagram for the case $a=\frac{1}{3}$, $m=0.9, e=1$ i.e. for $\rho=0.9$. Note that in this case $P_{4+}$ is infeasible, since $\tilde{p}_{+}>\rho$. The figure shows bistability with the equilibria $P_{2}^{C}$ and $P_{3}^{C}$.

\subsection{Stability}

The Jacobian matrix of (13) is

$$
J \equiv\left(\begin{array}{cc}
1-3 p^{2} & -a \\
-e f & m(1-2 f)-e p
\end{array}\right)
$$

At the origin, the eigenvalues are 1 and $m$, so that it is unstable. The point $P_{2}^{C}$ is stable if and only if

$$
\rho<1
$$

having eigenvalues -2 and $m-e$. At equilibrium $P_{3}^{C}$ instead the system reduces only to one logistic equation and on the axis $p=0$ this equilibrium is stable. Furthermore, if we consider the point $\left(\epsilon, f_{0}\right)$ with $\epsilon>0$ arbitrarily small and $f_{0}=1+\eta$, also with $\eta \in \mathbf{R}$ small, then we find that at this point $\frac{d}{d t} p=\epsilon\left(1-\epsilon^{2}\right)-a(1+\eta)=-a+O(\eta)+O(\epsilon)<0$, while $\frac{d}{d t} f=$ $m f_{0}\left(1-f_{0}\right)-e f_{0} \epsilon=-(1+\eta)(m \eta+e \epsilon)$ so that the sign depends on how relatively close to zero are the quantities $\eta$ and $\epsilon$. But the first inequality shows that $p$ decreases, so that ultimately $P_{3}^{C}$ is approached. Therefore it is locally asymptotically stable.

For the interior equilibria, we find that quantities needed by the Routh- 


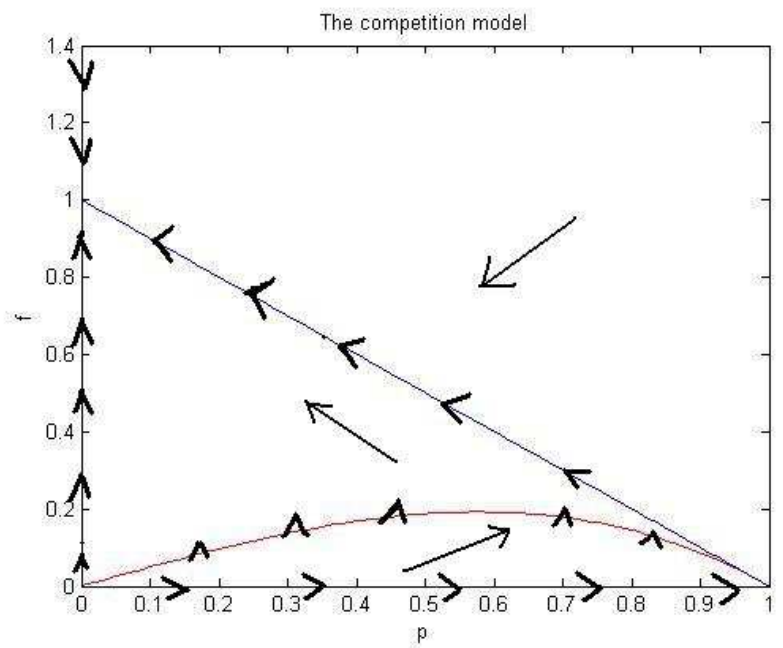

Figure 5: Competition model (14). Phase plane diagram for the case $a=2$, $m=1, e=1$ i.e. for $\rho=1$.

Hurwitz conditions become

$$
\begin{gathered}
(\mathrm{c}):-\operatorname{tr} J\left(P_{4 \pm}\right)=3 \widetilde{p}_{ \pm}^{2}-e \widetilde{p}_{ \pm}+m-1, \\
(\mathrm{~d}): \operatorname{det}\left(J\left(P_{4 \pm}\right)\right)=3 m \widetilde{p}_{ \pm}^{2}-a e-m .
\end{gathered}
$$

Let the roots of the quadratic associated to (c) be denoted by $p^{ \pm}=\frac{1}{6}(e \pm \Delta)$, where $\Delta=e^{2}+12(1-m)$. If $\Delta<0$, we have $-\operatorname{tr} J\left(P_{4 \pm}\right)>0$ always. In the opposite case the solutions of the inequality will lie outside the interval of the roots, namely in $\left[0, p^{-}\right] \cup\left[p^{+}, \infty\right)$ for $m \geq 1$ and in $\left[p^{+}, \infty\right)$ conversely for $m<1$. Instead $(\mathrm{d})$ holds for $\widetilde{p}_{ \pm}$in $[\bar{p}, \infty)$. Since $\widetilde{p}_{-}<\bar{p}$, the smaller root $\widetilde{p}_{-}$ will never satisfy this inequality and therefore the corresponding equilibrium $P_{4-}$ will always be unstable.

Combining the results for (c) and (d), both Routh-Hurwitz conditions will be satisfied for $\widetilde{p}_{+}$in some cases only, summarized in the following result.

Theorem 2. The interior equilibria of system (14), feasible if (15) holds, are characterized as follows: $P_{4-}$ when feasible, is always unstable. Furthermore

- for $\Delta<0$ the point $P_{4+}$ is a stable equilibrium;

- for $\Delta>0$ and $m<1$ the interior equilibrium $P_{4+}$ exists and is stable if $\widetilde{p}>\max \left\{\bar{p}, p^{+}\right\}$

- for $\Delta>0$ and $m>1$ the interior equilibrium $P_{4+}$ exists and is stable if $\widetilde{p}>\max \left\{p^{-}, \bar{p}, p^{+}\right\}$and $\bar{p}<\widetilde{p}<p^{-}$. 


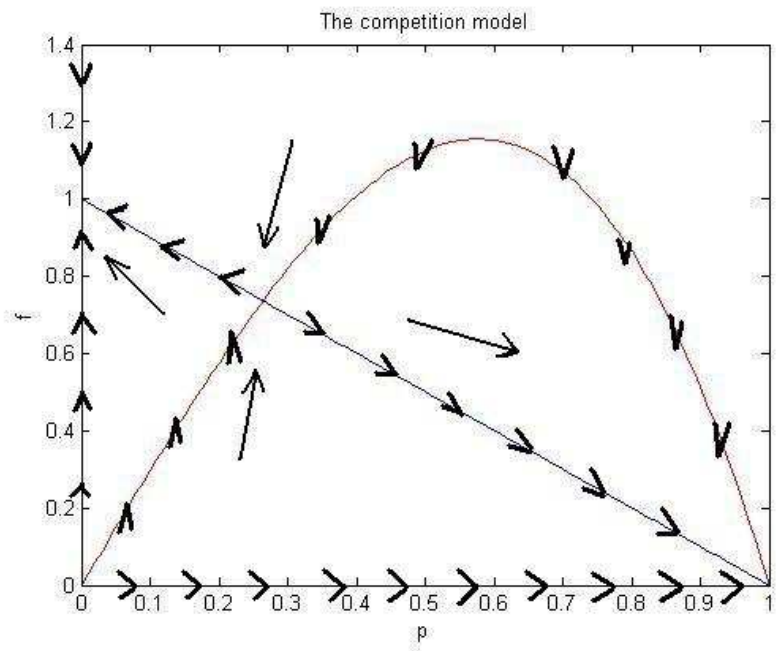

Figure 6: Competition model (14). Phase plane diagram for the case $a=\frac{1}{3}$, $m=1, e=1$ i.e. for $\rho=1$.

More precisely, for $m>1$ there can be either two equilibria or none, see respectively Figures 8 and 7 below. In the latter case, $P_{3}^{C}$ is always stable. In the former, $P_{4-}$ is a saddle and there is bistability, $P_{3}^{C}$ and $P_{4+}$ are both stable equilibria. For $\rho<1, P_{4-}$ is again a saddle, the model exhibits bistability, but in this case with the two boundary equilibria $P_{3}^{C}$ and $P_{2}^{C}$, Figure 4 , since $P_{4+}$ is infeasible. Therefore, in general, apart from the circumstance of $P_{4+}$ being feasible, the principle of competitive exclusion holding for the classical model, maintains its validity in the new model as well.

The borderline case of $\rho=1$ can have a single stable equilibrium, Figure 5 , but it can also exhibit bistability, Figure 6 , depending on whether $a>1$ or $a<1$.

Bistability for $\Delta>0$ and $m>1$ is however a novel feature of the system (14), compared with the classical competition model. In fact, depending on the initial condition, either only the population $f$ survives, at equilibrium $P_{3}^{C}$, or both populations survive, at the stable interior equilibrium. The herd behavior then acts as a factor which could allow the survival of the individualistic population, a counterintuitive result.

\section{The predator-prey model}

In this context, $F$ denotes the predator population and $R$ the prey, the latter exhibiting a highly socialized behavior, living in herds, the weaker individuals being kept at the center of their herd for defensive purposes. If the prey 


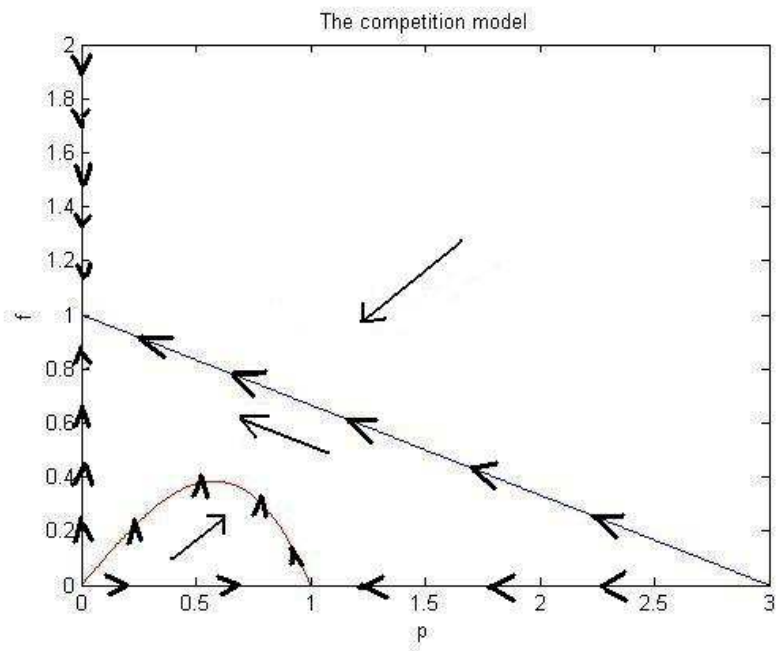

Figure 7: Competition model (14) with no interior equilibria. Phase plane diagram for the case $a=1, m=9, e=3$ i.e. for $\rho=3$. Condition (15) cannot be satisfied since here $\bar{p}=\frac{2}{3}$ and therefore $\bar{p}^{3}=\frac{8}{27}<\frac{1}{2}=\frac{1}{2} a$.

experience intraspecific competition and represent the only food source for the predators, the system becomes

$$
\begin{aligned}
\frac{d}{d t} R(t) & =r\left(1-\frac{R(t)}{K}\right) R(t)-a \sqrt{R(t)} F(t), \\
\frac{d}{d t} F(t) & =-\widetilde{m} F(t)+a \widetilde{e} \sqrt{R(t)} F(t)
\end{aligned}
$$

with $r$ denoting the prey net reproduction rate and $K$ their carrying capacity, $\widetilde{e}$ the conversion coefficient and $\widetilde{m}$ and $a$ the predators' mortality and hunting rates.

Rescaling as done earlier in Section 3.4 leads to

$$
\begin{aligned}
\frac{d}{d t} p(t) & =\left(1-p^{2}\right) p-f, \\
\frac{d}{d t} f(t) & =(e p-m) f=e f(p-\rho) .
\end{aligned}
$$

\subsection{Equilibria}

We find the points

$$
P_{1}^{P}=(0,0), \quad P_{2}^{P}=(1,0), \quad P_{4}^{P} \equiv\left(p^{*}, f^{*}\right)=\left(\rho, \rho\left(1-\rho^{2}\right)\right),
$$




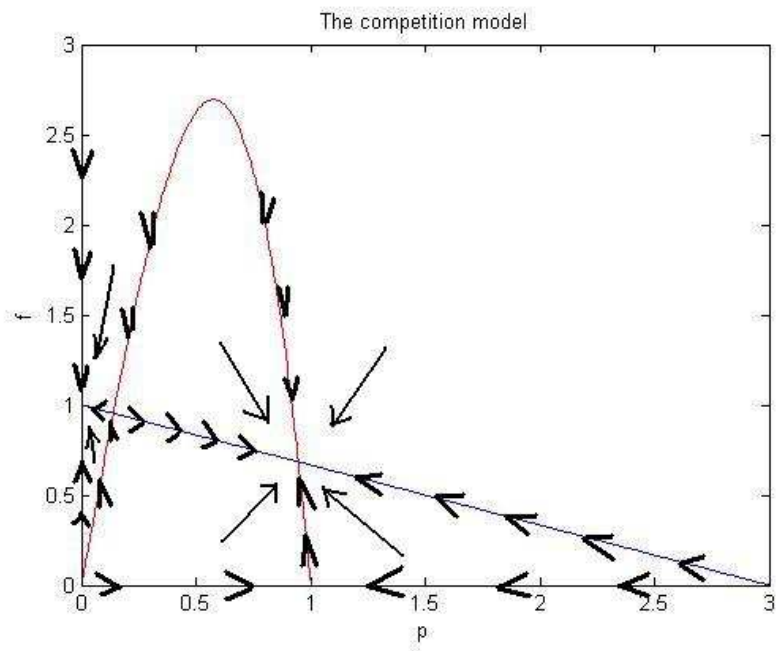

Figure 8: Competition model (14) with two interior equilibria. Phase plane diagram for the case $a=\frac{1}{7}, m=9, e=3$ i.e. for $\rho=3$. Condition (15) is satisfied since now $\bar{p}=\frac{22}{63}$ and therefore $\bar{p}^{3}=0.2064>0.0714=\frac{1}{2} a=\frac{1}{14}$.

the first two being inconditionally feasible. $P_{4}^{P}$ requires nonnegativity of the predator population, giving the feasibility condition

$$
\rho<1 .
$$

Note further that in this case the point $P_{3}^{P}=(0,1)$ is not an equilibrium, as for the case of obligated mutualism and contrary to the other symbiotic and competing models.

\subsection{Stability}

The system (20) has the Jacobian

$$
J \equiv\left(\begin{array}{cc}
1-3 p^{2} & -1 \\
e f & e p-m
\end{array}\right) .
$$

At the origin the eigenvalues are 1 and $-m$ for which $P_{1}^{P}$ is unstable. At $P_{2}^{P}$ they are -2 and $e-m$. The stability condition for $P_{2}^{P}$ then reduces to the opposite of (18), namely of what happens in the competing case, and also to the opposite of the feasibility condition for $P_{4}^{P}$ in this system, (21), namely

$$
\rho>1 .
$$


For the Routh-Hurwitz conditions for the interior equilibrium we find

$$
-\operatorname{tr} J\left(P_{4}^{P}\right)=3 \rho^{2}-1, \quad \operatorname{det}\left(J\left(P_{4}^{P}\right)\right)=m\left(1-\rho^{2}\right) .
$$

The conditions are satisfied respectively for $\rho>(\sqrt{3})^{-1}$ and $\rho<1$.

The above considerations can be summarized as follows. The origin, $P_{1}^{P}$, is always unstable, and the results for the equilibria $P_{2}^{P}$ and $P_{4}^{P}$ give several situations summarized in Table 1 . Note that $P_{2}^{P}$ is stable if and only if $P_{4}^{P}$ is infeasible, compare (23) and (21).

Table 1: Equilibria of system (19)

\begin{tabular}{|c|c|c|c|}
\hline Condition & $P_{2}^{P}$ & $P_{4}^{P}$ & bifurcation \\
\hline$\rho>1$ & asymptotically stable & infeasible & \\
$\rho=1$ & & & \\
$\frac{1}{\sqrt{3}}<\rho<1$ & unstable & asymptotically stable & \\
$\rho=\frac{1}{\sqrt{3}}$ & unstable & & Hopf \\
$0<\rho<\frac{1}{\sqrt{3}}$ & unstable & unstable & \\
\hline
\end{tabular}

\subsubsection{System's behavior in terms of $\rho$}

We investigate the system's behavior assessing the $\omega$-limit sets of the dynamical system in terms of $\rho$, by drawing its nullclines in the phase plane.

\section{The case $\rho>1$}

$P_{2}^{P}$, the predator-free equilibrium, is locally asymptotically stable and the coexistence equilibrium is infeasible, since the nullclines intersect for $f<0$, see Figure 9. The system's trajectories are easily shown to be bounded. Indeed let

$$
f^{+} \equiv f\left(\frac{1}{\sqrt{3}}\right)=\frac{2}{3 \sqrt{3}}
$$

be the maximum value of the nullcline for $p$. Then the set $T=\{(p, f): \rho \geq$ $\left.p \geq(\sqrt{3})^{-1}, f \leq f^{+}\right\}$, is a positively invariant set of (19), Figure 10. Note that the trajectories enter into the set $T$ on the vertical line $p=\rho$, since they 


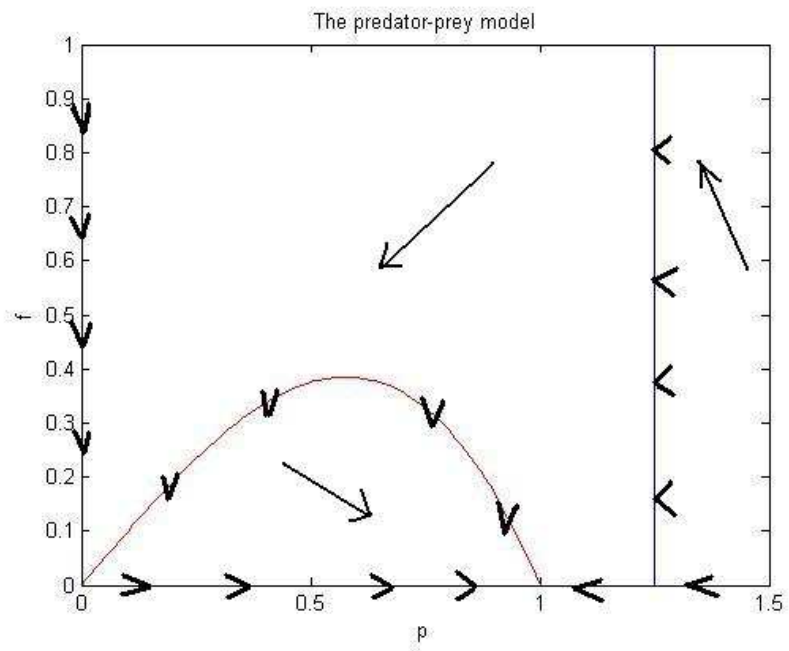

Figure 9: Predator-prey model (20). Phase plane diagram for the case $m=$ $1.25, e=1$ i.e. for $\rho=1.25$.

are oriented down and to the left. Trajectories, see Figure 9, move similarly down and to the left on the horizontal line $f=f^{+}$, which is not shown. On the vertical line $p=(\sqrt{3})^{-1}$ instead trajectories move down and to the right.

Before proceeding, we subdivide the first quadrant into a few subsets, which will help in the analysis. Regions I-IV are defined as follows:

$$
\begin{array}{r}
I=\{(p, f): p \geq \rho\}, \\
I I=\left\{(p, f): p \leq \rho f \geq f^{+}\right\}, \\
I I I=\left\{(p, f): p \leq(\sqrt{3})^{-1}, p\left(1-p^{2}\right) \leq f \leq f^{+}\right\}, \\
I V=\left\{(p, f): p \leq(\sqrt{3})^{-1}, p\left(1-p^{2}\right) \geq f\right\} .
\end{array}
$$

These regions are graphically depicted in Figure 10.

Lemma 1. Starting from region I, at some suitable time $t_{2}>0$ the prey population attains the level $p\left(t_{2}\right)=\rho$. Thus trajectories originating in region I enter either into region II, Figure 10, or into the set $T$.

Proof. Consider the initial condition $\left(p_{0}, f_{0}\right)$ for the solution of $(20)$. Here $p_{0}>\rho>1$. Thus the second equation in (20) gives a nonnegative derivative, $\frac{d f}{d t} \geq 0$ so that $f(t) \geq f_{0}$ follows. In view of the inequality $1-p_{0} \leq 0$, from the first equation in $(20)$ we find $\frac{d}{d t} p(t) \leq-f \leq-f_{0}$ so that $p$ decreases from its initial value $p_{0}$ as $t$ grows, until eventually the inequality $p \geq 1$ ceases to hold. Integrating we find

$$
p(t) \leq-f_{0} t+p_{0}
$$




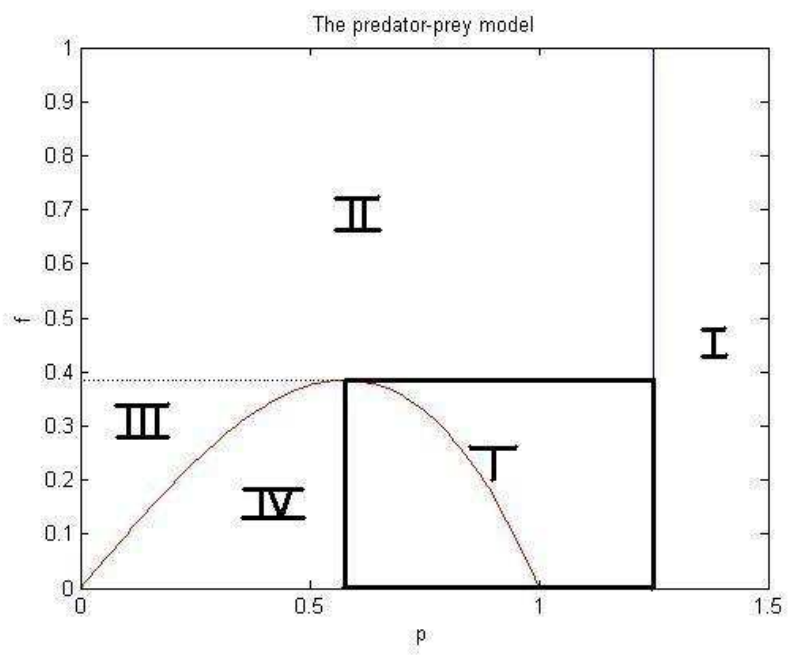

Figure 10: Invariant set $T$ and regions I-IV.

Let $t_{1}$ be the time at which $p\left(t_{1}\right)=\rho$, namely $t_{1}=\frac{p_{0}-\rho}{f_{0}}$. From the inequality (24) evaluated at time $t_{1}$, we find

$$
p\left(t_{1}\right) \leq \rho
$$

Now two alternatives are possible. Either $p(t) \geq \rho$ for every $t \in\left[0, t_{1}\right]$, or in $\left[0, t_{1}\right]$ there is a $t^{*}$ for which $p\left(t^{*}\right)<\rho$. In the former case it follows $p\left(t_{1}\right) \geq \rho$ which combined with (25) gives $p\left(t_{1}\right)=\rho$, i.e. we take $t_{2}=t_{1}$. In the second case the continuity of the function $p(t)$ ensures the existence of a $t_{2} \in\left[0, t^{*}\right]$ such that $p\left(t_{2}\right)=\rho$. There are two possible cases now. Either $f\left(t_{2}\right) \geq f^{+}$, in which case the trajectory enters region II, or $f\left(t_{2}\right) \leq f^{+}$in which case it enters the positively invariant set $T$.

Lemma 2. Starting from region II, at some suitable time $t_{2}>0$ the predator population attains the level $f\left(t_{2}\right)=f^{+}$for $p>(\sqrt{3})^{-1}$. Thus trajectories originating in region II enter either into region III, Figure 10, or into the set $T$.

Proof. Consider the initial condition $\left(p_{0}, f_{0}\right)$ for the solution of $(20)$, assuming $p_{0}<\rho, f_{0}>f^{+}$. Since $\frac{d p}{d t} \leq 0$, it follows $p \leq p_{0}$. From the second equation in (20) we have $\frac{d f}{d t}=e f(p-\rho) \leq e f\left(p_{0}-\rho\right) \equiv-C f, C>0$. Integrating, $f(t) \leq f_{0} \exp [-C t] \rightarrow 0$ as $t \rightarrow \infty$. Hence proceeding as in Lemma 1, for some $t_{2}>0, f\left(t_{2}\right)=f^{+}$. At this time, either $p\left(t_{2}\right) \geq(\sqrt{3})^{-1}$, for which the trajectory enters into the set $T$, or $p\left(t_{2}\right) \leq(\sqrt{3})^{-1}$, in which case the trajectory enters region III. 
Lemma 3. All trajectories originating in region III enter into region IV, Figure 10.

Proof. Take the initial condition $\left(p_{0}, f_{0}\right)$ in region III, so that $p_{0}<$ $(\sqrt{3})^{-1}<\rho, p\left(1-p^{2}\right)<f_{0} \leq f^{+}$. For the solution of (20) we have $\frac{d f}{d t}<0$. Furthermore $\frac{d f}{d t} \leq$ ef $\left((\sqrt{3})^{-1}-\rho\right)=-K f, K>0$. Integrating, $f(t) \leq$ $f_{0} \exp (-K t)$. Thus for every $\epsilon>0$ there are two cases: either for all $t>0$ $f(t) \geq p(t)\left(1-p^{2}(t)\right)+\epsilon$ so that $\frac{d p}{d t}<-\epsilon$ from which integrating we find $p(t) \leq-\epsilon t+p_{0}$ implying then the existence of some $t^{*}$ for which $p\left(t^{*}\right)=$ 0 . But this is not possible because then through the point $\left(p\left(t^{*}\right), f\left(t^{*}\right)\right)=$ $\left(0, f\left(t^{*}\right)\right)$ there would be two system's trajectories, the one through $\left(p_{0}, f_{0}\right)$ and the coordinate axis $p=0$, contradicting the existence and uniqueness theorem. In the second case, for every $\epsilon>0$ there is some $\bar{t}>0$ for which $f(\bar{t})-p(\bar{t})\left(1-p(\bar{t})^{2}\right)<\epsilon$, which implies $f(\bar{t}) \leq p(\bar{t})\left(1-p(\bar{t})^{2}\right)$ and therefore $\frac{d p}{d t} \geq 0$, inequality stating that the trajectory has entered region IV.

Lemma 4. All trajectories originating in region IV enter into the positive invariant set $T$, Figure 10.

Proof. Here we have $\frac{d p}{d t} \geq 0$, for which $p$ grows. Hence $\frac{d p}{d t}=p\left(1-p^{2}\right)-$ $f \geq p \geq p_{0}$ and integrating $p(t) \geq p_{0}(t+1)$. It follows that for some $t_{1}$, $p\left(t_{1}\right) \geq(\sqrt{3})^{-1}$, and reasoning as in Lemma 2 , it follows that there is $t_{2}$ such that $p\left(t_{2}\right)=(\sqrt{3})^{-1}$, i.e. the trajectory enters into the set $T$.

Lemma 5. No closed orbits exist in $T$.

Proof. Considering the expression

$$
\begin{array}{r}
\frac{\partial}{\partial p}\left[\frac{d p}{d t}\right]+\frac{\partial}{\partial f}\left[\frac{d f}{d t}\right]=\frac{\partial}{\partial p}\left[\left(p\left(1-p^{2}\right)-f\right)\right]+\frac{\partial}{\partial f}[e f(p-\rho)] \\
=1-3 p^{2}+e(p-\rho)<0,
\end{array}
$$

since by construction in $T$ we have $\rho>p>(\sqrt{3})^{-1}$, we find that it is one-signed in $T$. Hence no closed orbits exist in $T$, by Dulac's theorem.

Combining the results of Lemmas 1-4, all the trajectories ultimately are confined to the invariant set $T$. By Lemma 5 no periodic orbits exist in $T$. Since $P_{2}^{P}$ is the only possible equilibrium in $T$, all the system's trajectories must approach it. The following result summarizes these considerations.

Theorem 3. In the case $\rho>1, P_{2}^{P}$ is globally asymptotically stable.

The vertical nullcline moves to the left when $\rho$ decreases toward the value $\rho=1$. At this value the two nullclines intersect at $P_{2}^{P} \equiv P_{4}^{P}$. Past this value, 
the coexistence equilibrium $P_{4}^{P}$, i.e. the nullclines' intersection, becomes feasible while $P_{2}^{P}$ becomes unstable, showing the transcritical bifurcation.

The case $\frac{1}{\sqrt{3}}<\rho<1$

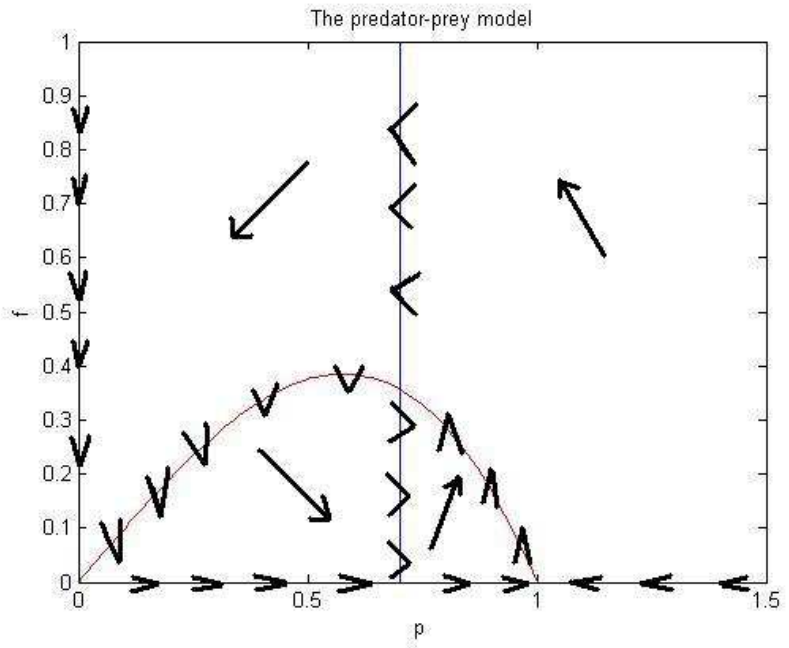

Figure 11: Predator-prey model (20). Phase plane diagram for the case $m=0.7, e=1$ i.e. for $\rho=0.7$.

Here, in addition or as replacement of regions previously defined, we also introduce the following ones:

$$
\begin{array}{r}
I a=\{(p, f): p \geq 1\}, \\
I b=\{(p, f): 1 \geq p \geq \rho, f \geq \bar{f}(p)\}, \\
I I b=\left\{(p, f): p \leq \rho, p\left(1-p^{2}\right) \leq f \leq f^{+}\right\}, \\
V=\left\{(p, f): \rho \leq p \leq 1, p\left(1-p^{2}\right) \geq f\right\}, \\
V I=\left\{(p, f): \rho \geq p \geq(\sqrt{3})^{-1}, p\left(1-p^{2}\right) \geq f\right\},
\end{array}
$$

where $\bar{f}(p)$ denotes the system's trajectory through the point $\left((\sqrt{3})^{-1}, f^{+}\right)$ These regions are graphically depicted in Figure 12.

Table 1 shows that $P_{4}^{P}$ is stable, so that the trajectories will approach this equilibrium, although this clearly cannot be seen from the phase plane picture alone, see for instance Figure 11 and compare it with Figure 17.

Here the nullclines $f=p\left(1-p^{2}\right)$ and $p=\rho$ intersect at the feasible coexistence equilibrium $P_{4}^{P}$.

Remark. By a procedure similar to Lemma 1, it is seen that trajectories from region Ia will enter into region Ib. 


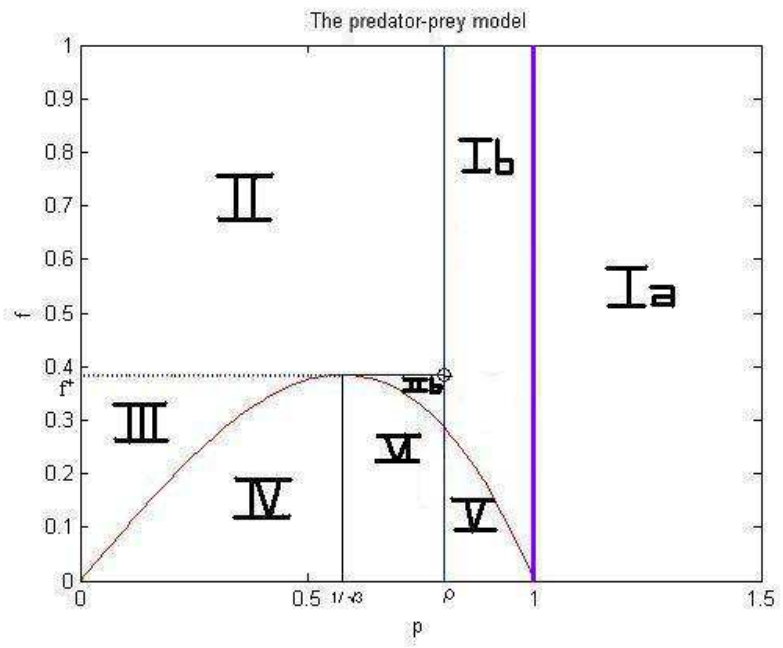

Figure 12: Predator-prey model (20). Regions Ia, Ib, II, IIb, III, IV, V, VI.

We now construct a positively invariant set. But for this task we need to integrate backwards, so taking $t=-s$ and $\bar{p}(s)=p(-t), \bar{f}(s)=f(-t)$, the system (20) becomes

$$
\begin{array}{r}
\frac{d}{d s} \bar{p}(s)=\bar{f}(s)-\bar{p}(s)\left(1-\bar{p}(s)^{2}\right), \\
\frac{d}{d s} \bar{f}(s)=e \bar{f}(s)(\rho-\bar{p}(s)) .
\end{array}
$$

Lemma 6. Moving backwards, the trajectory starting at $\left(p_{0}, f_{0}\right) \equiv$ $\left(\rho, f^{+}\right)$in a finite time either hits the vertical line $\bar{p}=1$, at level say $\bar{f}=f^{-}$, or crosses into region $V$.

Proof. Either for every $\bar{p}$ such that $1>\bar{p}>\rho$, we have at all times $\bar{f}-\bar{p}\left(1-\bar{p}^{2}\right)>L$, for an arbitrary $L>0$, or there is an $s^{*}$ such that $\bar{f}\left(s^{*}\right)=\bar{p}\left(s^{*}\right)\left(1-\bar{p}^{2}\left(s^{*}\right)\right)$. In the last case the trajectory enters region $\mathrm{V}$. In the former, we have $\bar{p}^{\prime}>L$ from which integrating $\bar{p}(s)>L s+p_{0}$ and then there is $s_{1}$ for which $p\left(s_{1}\right) \geq 1$ as claimed. Set then $f^{-}=\bar{f}\left(s_{1}\right)$.

In the former case it follows that a positive invariant set $E_{1}$ can be constructed by taking the $p$ axis for $(\sqrt{3})^{-1} \leq \bar{p} \leq 1$, the vertical lines $p=(\sqrt{3})^{-1}$, up to height $f^{+}$, and $p=1$, up to height $f^{-}$, the horizontal segment at height $f=f^{+}$for $(\sqrt{3})^{-1}<p<\rho$, and the trajectory joining the points $\left(\rho, f^{+}\right)$and $\left(1, f^{-}\right)$just constructed in Lemma 6 , see Figure 13.

Lemma 7. Moving backwards, the trajectory entering region $\mathrm{V}$ from Lemma 6 enters region VI in a finite time. 


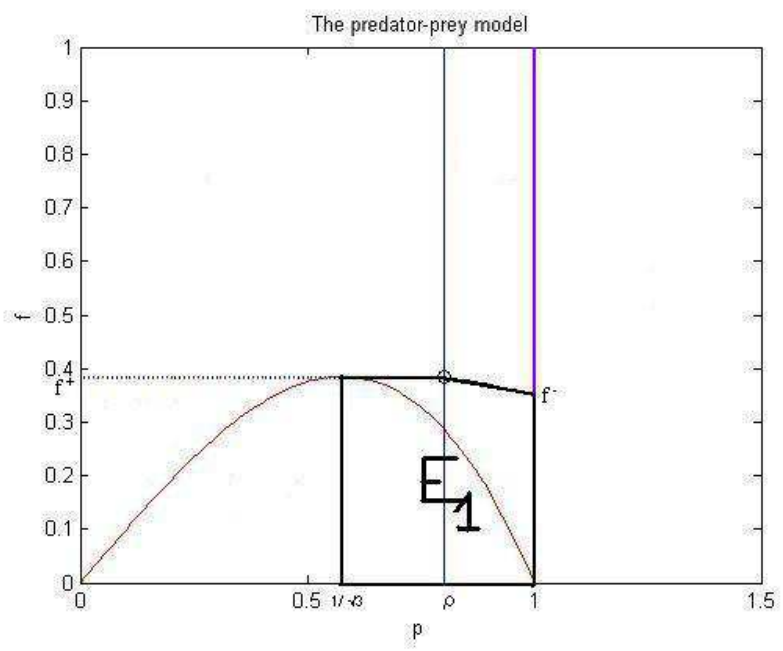

Figure 13: Invariant set $E_{1}$.

Proof. Let us assume that for every $s$ we have $\bar{p}(s)-\rho>H$ for some $H>$ 0 . Then $\bar{f}^{\prime}<-e H \bar{f}$ from which, integrating, we have $\bar{f}<\bar{f}_{0} \exp (-e H s)$. It follows that $\bar{p}^{\prime} \leq f_{0} \exp (-e H s)-\bar{p}\left(1-\bar{p}^{2}\right) \leq f_{0} \exp (-e H s)-\rho\left(1-\bar{p}_{0}^{2}\right)$, since $\bar{p}>\rho$ and $1-\bar{p}>1-\bar{p}_{0}$. Integrating once more $\bar{p}(s) \leq(e H)^{-1} f_{0}[1-$ $\exp (-e H s)]+\bar{p}_{0}-\rho\left(1-\bar{p}_{0}^{2}\right) s$. Clearly $p(s) \rightarrow-\infty$ as $s \rightarrow+\infty$ so that for some $\bar{s}$, we have $\bar{p}(\bar{s})<\rho$, contradicting the assumption. Hence there is $\widehat{s}$ such that $\bar{p}(\widehat{s}) \leq \rho$, i.e. the trajectory enters region VI.

Lemma 8. Moving backwards, the trajectory entering region VI from Lemma 7 either crosses the vertical line $p=(\sqrt{3})^{-1}$ at height say $f_{+}$, or it enters region $\mathrm{IIb}$ in a finite time.

Proof. In this region, since $\bar{p}^{\prime}<0$, either we find $\bar{p}(\widetilde{s}) \leq(\sqrt{3})^{-1}$ for some $\widetilde{s}>0$, in which case the first conclusion holds, or for all $s>0$ and for some $M>0, \bar{p}(s) \geq(\sqrt{3})^{-1}+M$ holds. Since $\bar{f}^{\prime}=e \bar{f}(\rho-\bar{p})>e \bar{f}\left(\rho-\bar{p}_{0}\right) \equiv N f>0$ then it follows for that for some $\bar{s}$ we find $f(\bar{s}) \geq f_{0} \exp (N \bar{s}) \geq 2(3 \sqrt{3})^{-1}=$ $\max \bar{p}\left(1-\bar{p}^{2}\right)$, or in other words $\bar{f}$ grows larger than $\bar{p}\left(1-\bar{p}^{2}\right)$. There is thus $s_{*}$ such that $\bar{f}\left(s_{*}\right)=\bar{p}\left(s_{*}\right)\left(1-\bar{p}\left(s_{*}\right)^{2}\right)$, for which then $\bar{p}^{\prime}\left(s_{*}\right)=0$. This implies that the trajectory enters region IIb.

In case the vertical line is crossed, we can define the positive invariant set $E_{2}$ as being bounded above by the segment $f=f^{+}$between the lines $p=(\sqrt{3})^{-1}$ and $p=\rho$, the trajectory constructed from point $\left(\rho, f^{+}\right)$joining it backwards with point $\left((\sqrt{3})^{-1}, f_{+}\right)$, the vertical segment $p=(\sqrt{3})^{-1}$ between $f=f_{+}$and $f=f^{+}$, see Figure 14 . 


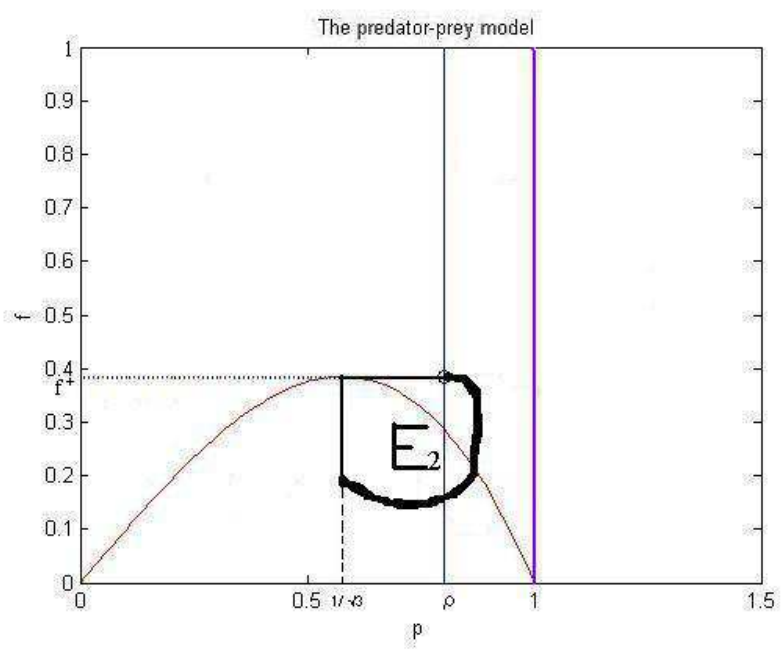

Figure 14: Invariant set $E_{2}$.

Lemma 9. Moving backwards, the trajectory entering region IIb from Lemma 8 in a finite time crosses first the horizontal line $f=f^{+}$and afterward the vertical line $p=\rho$ at a level $f_{*}>f^{+}$.

Proof. We backtrack from the point $\left(\bar{p}_{0}, \bar{f}_{0}\right) \equiv\left(\bar{p}_{0}, \bar{p}_{0}\left(1-\bar{p}_{0}^{2}\right)\right)$. Since $\bar{p}_{0}<\rho$, we find $\bar{f}^{\prime}-e \bar{f}\left(\rho-\bar{p}_{0}\right)>0$, so that $\bar{f}$ grows. Also, $\bar{p}^{\prime}>0$ and $\bar{p}$ grows as well, $\bar{p}>\bar{p}_{0}$. Integrating we find $\bar{f}(s)>\bar{f}_{0} \exp \left(e\left(\rho-\bar{p}_{0}\right) s\right)$. Hence for some $s^{+}$we find $\bar{f}\left(s^{+}\right)=f^{+}$and we can have either $p_{-} \equiv \bar{p}\left(s^{+}\right)<\rho$ or $\bar{p}\left(s^{+}\right) \geq \rho$. In the former case the horizontal line is hit first as claimed. The latter case implies then that the trajectory crosses first the vertical line $p=\rho$ but this must occur at the height $\bar{f}\left(s^{+}\right)<f^{+}$. This entails that traversing the trajectory forwards, the same would wind around the equilibrium point but going farther away from it, in other words it would behave as the equilibrium were locally asymptotically unstable, contradicting our former local stability analysis. Hence the first alternative must hold, implying that there is $s_{*}<s^{+}$ such that $\bar{p}\left(s_{*}\right)=\rho$, with $\bar{f}\left(s_{*}\right) \equiv f_{*}>f^{+}$.

Since the trajectory hits the horizontal line, the positively invariant set $E_{3}$ is bounded by the horizontal segment $f=f^{+}$for $p \in\left[p_{-}, \rho\right]$ and the trajectory originating at $\left(\rho, f^{+}\right)$and ending in $\left(p_{-}, f^{+}\right)$, see Figure 15 . Alternatively another invariant set $E_{4}$, can be constructed by bounding it by the whole trajectory originating from $\left(\rho, f^{+}\right)$, moving forwardly, and winding around the equilibrium point until it reaches the point $\left(\rho, f_{*}\right)$. The set is completed by the vertical segment joining these very same points. On the latter, being situated on the vertical line $p=\rho$, the flux points inward, see 
Figures 11 and 16.

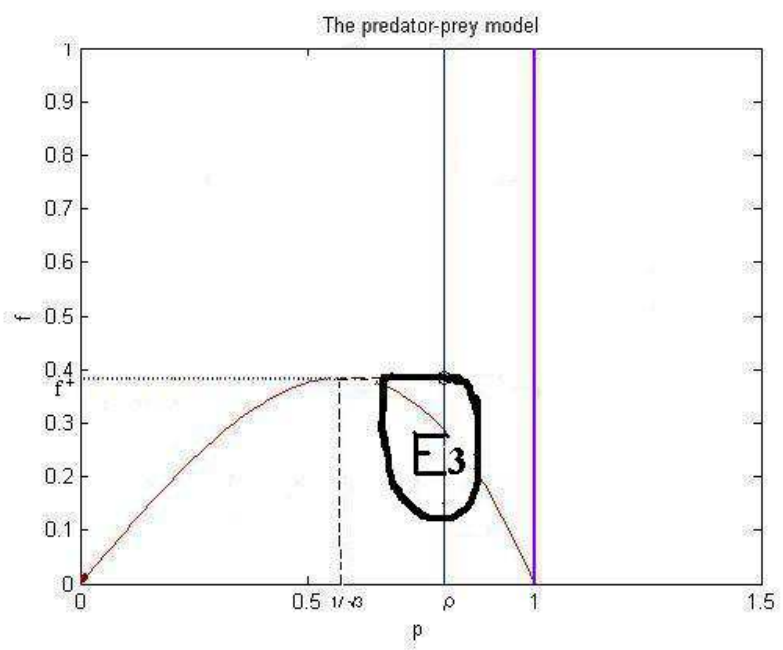

Figure 15: Invariant set $E_{3}$.

Lemma 10. In the positively invariant sets $E_{1}, E_{2}, E_{3}$, or possibly $E_{4}$, no periodic orbits exist.

Proof. Here we take $B(p, f)=f^{-1}$, and calculate

$$
\begin{aligned}
& \frac{\partial}{\partial p}\left[B(p, f)\left(p\left(1-p^{2}\right)-f\right)\right]+\frac{\partial}{\partial f}[B(p, f) e f(p-\rho)] \\
= & \frac{\partial}{\partial p}\left[\frac{p}{f}\left(1-p^{2}\right)-1\right]+\frac{\partial}{\partial f}[e(p-\rho)]=\frac{1}{f}\left(1-3 p^{2}\right)<0,
\end{aligned}
$$

in view of the fact that $(\sqrt{3})^{-1}<p$ in all these sets. Thus Dulac's theorem prevents the existence of periodic orbits.

Remark. Here the set $E_{1}$ cannot be constructed, we need to check what a trajectory does, namely whether it enters into the sets $E_{2}, E_{3}$ and $E_{4}$. Consider any trajectory originating at $(1, \widetilde{h})$, with $\widetilde{h}>f_{-}$. It can be shown either to enter these sets, or to wind around them as follows, by using the statements of Lemmas 6-9, this time moving forwards. In fact the trajectory will traverse region Ib, II, III, IV, VI and V, unless entering into set $E_{2}$ and then since it cannot cross itself, it must wind around these sets, or enter into set $E_{4}$ or $E_{3}$. In case these sets are never entered, the trajectory will approach their boundary arbitrarily closely. It follows that the boundary of these regions will itself be a closed orbit of the system. But this contradicts Lemma 10, and therefore the trajectory must ultimately enter one of the sets $E_{2}, E_{3}$ or $E_{4}$. 


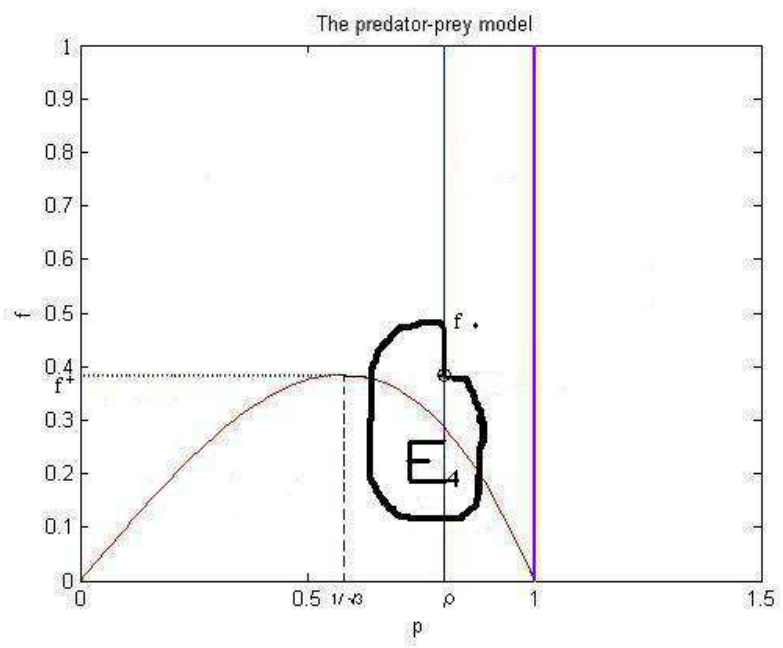

Figure 16: Invariant set $E_{4}$.

Let $E$ denote any one of the above constructed positively invariant sets. Then since the equilibrium $P_{4}^{P}$ is locally asymptotically stable and being the only equilibrium in $E$ which is an attracting set containing no closed orbits, it must also be globally asymptotically stable.

In summary we have the following theorem.

Theorem 4. In the case $\frac{1}{\sqrt{3}}<\rho<1, P_{4}^{P}$ is globally asymptotically stable.

As $\rho$ decreases, the vertical nullcline moves again to the left and it finally crosses the vertex of the parabola, which represents the second nullcline, for the value $\rho=\sqrt{3}^{-1}$. The Jacobian eigenvalues become purely imaginary, giving the Hopf bifurcation: sustained oscillations then occur.

The case $0<\rho<\frac{1}{\sqrt{3}}$

Here we also need to define two additional regions. Let $\tilde{f}(p)$ denote the system's trajectory through the point $(1, h)$, with $h \geq f^{+}$, reaching the vertical line $p=\rho$ at height $h^{*}$, i.e. at the point $\left(\rho, h^{*}\right)$.

$$
\begin{aligned}
I I a= & \{(p, f): 1 \geq p \geq \rho f \geq \tilde{f}(p)\}, \\
& I I c=\left\{(p, f): p \leq \rho f \geq h^{*}\right\},
\end{aligned}
$$

These regions are graphically depicted in Figure 18. 


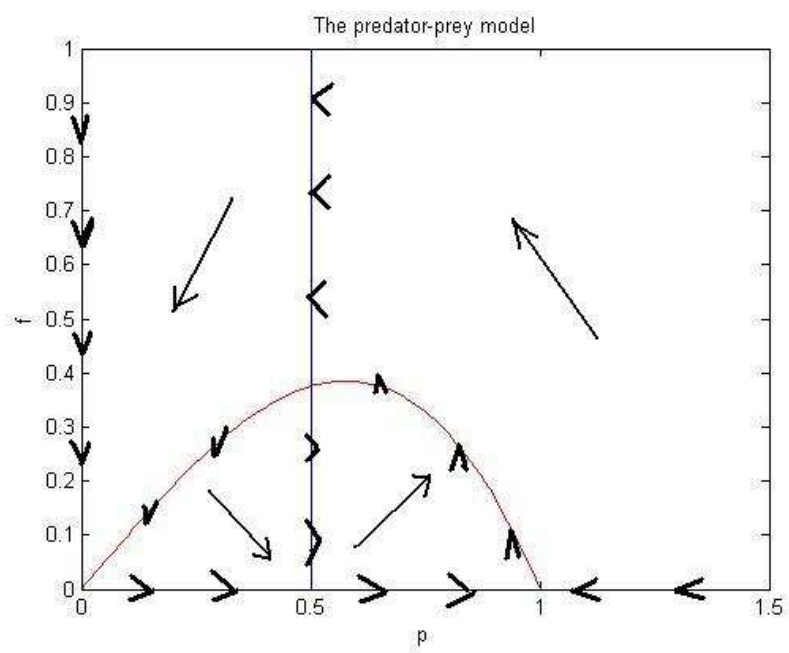

Figure 17: Predator-prey model (20). Phase plane diagram for the case $m=0.5, e=1$ i.e. for $\rho=0.5$.

Since from Table 1 all the possible system's equilibria become unstable, we need to determine in this situation what the system's $\omega$-limit sets are. To this end we study trajectories originating on or to the right of the vertical line $p=1$, see Figure 17 .

We construct a set $\Sigma$, as follows. Start by considering the vertical line through $p=1$ up to a point with height $h \geq f^{+}$; from there we follow the trajectory with initial condition $(1, h)$ until it intercepts the vertical nullcline, say at the point $\left(\rho, h^{*}\right)$. To complete $\Sigma$, we take the horizontal line $f=h^{*}$ from this interception up to the vertical axis together with the two segments on the coordinate axes, as depicted in Figure 18.

Lemma 11. From region Ia trajectories enter either into $\Sigma$ or into region IIa.

Proof. Here $\frac{d f}{d t}>0$ so that $f \geq f_{0}$, and $1-p<0$ so that $\frac{d p}{d t}=$ $p\left(1-p^{2}\right)-f<-f_{0}$. Integrating $p(t) \leq-f_{0} t+p_{0}$ so that there is some $t_{*}$ for which $p\left(t_{*}\right) \leq 1$. Then either $f\left(t_{*}\right)<h$, and trajectory enters into $\Sigma$, or $f\left(t_{*}\right)>h$ in which case it enters region IIa as claimed.

Lemma 12. From region IIa trajectories enter into region IIc.

Proof. In this case $\frac{d f}{d t}-e f(p-\rho)>e f_{0} p>e f_{0} \rho \equiv Q$ since $p>\rho$ and $\frac{d f}{d t}>0$, for which $f>f_{0}$. Then $f(t) \geq Q t+f_{0}$ and substituting into the equation for $p$, we get $\frac{d p}{d t}<p\left(1-p^{2}\right)-Q t-f_{0}<p_{0}\left(1+p_{0}\right)-Q t-f_{0}$, since $1-p<1, p<p_{0}$. But the term on the right tends to $-\infty$ as $t \rightarrow \infty$, so there is $\tilde{t}$ for which $p(\tilde{t}) \leq \rho$. 


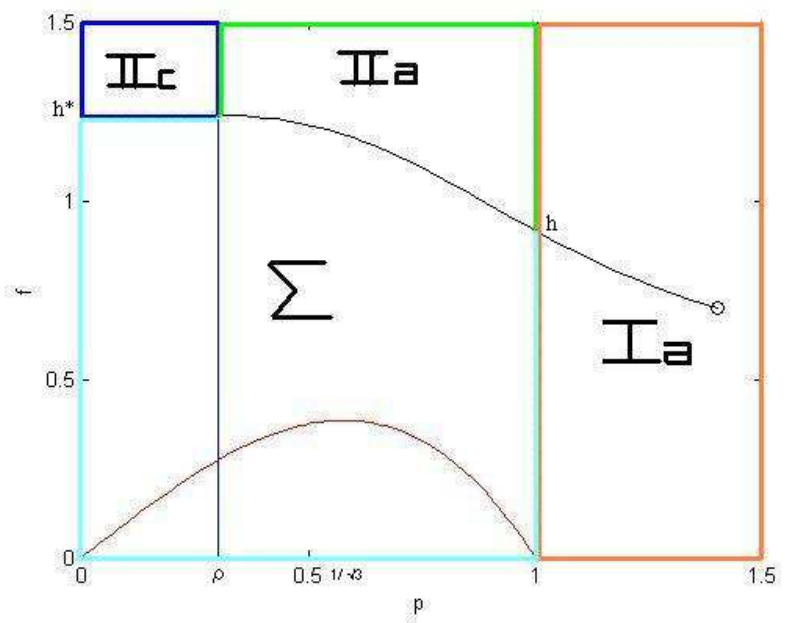

Figure 18: Predator-prey model (20). Regions IIa, IIc and positively invariant set $\Sigma$ for $\rho<(\sqrt{3})^{-1}$.

Lemma 13. From region IIc trajectories enter into $\Sigma$.

Proof. From $\frac{d p}{d t}<0, \frac{d f}{d t}<0$, we have $p<p_{0}, f<f_{0}$. Taking $p_{0}<\rho$, we find $\frac{d f}{d t}<e f\left(p_{0}-\rho\right) \equiv-R$, with $R>0$. Integrating, $f(t) \leq-R t+f_{0}$, so that there is $\hat{t}$ for which $f(\hat{t}) \leq h^{*}$, where $\left(\rho, h^{*}\right)$ by construction is the point crossed by the trajectory originating in $(1, h)$. Thus from $\left(p_{0}, f_{0}\right)$ in region IIc the trajectory enters into the set $\Sigma$.

Lemma 14. The set $\Sigma$ is positively invariant.

Proof. In fact the axes and the trajectory joining $(1, h)$ and $\left(\rho, h^{*}\right)$ cannot be crossed. On the vertical segment $p=1$ the flows points inwards, as well as on the horizontal segment.

Theorem 5. $\Sigma$ contains any possible trajectory of the system.

Proof. Combine the results of Lemmas 11, 12, 13 and 14.

Finally, to show the existence of a limit cycle, it is straightforward to use the Poincaré-Bendixson theorem, since the coexistence equilibrium in this case is unstable. Our simulations reported in Figure 19 show it explicitly. The following result summarizes our considerations.

Theorem 6. For the case $0<\rho<\frac{1}{\sqrt{3}}$, the system (20) possesses a limit cycle. 
Remark. Combining Theorems 5 and 6, all system's trajectories are attracted by this limit cycle.
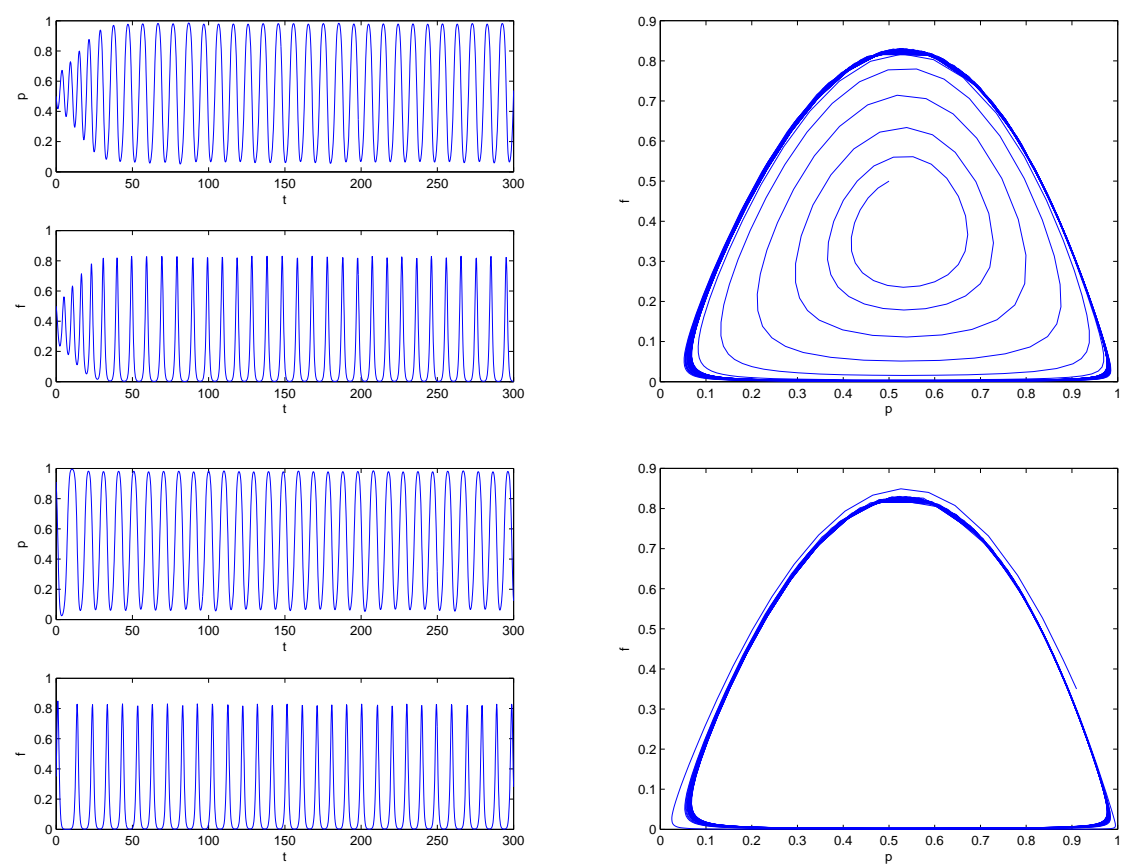

Figure 19: Predator-prey model (20). Simulations for the parameter values $e=3.74, m=2$, which imply $\rho=0.5348$. Left: solutions as functions of time. Right: phase plane plots showing the limit cycle whose existence is proven by the Poincaré-Bendixson theorem, with initial conditions $(0.5 ; 0.5)$ inside the limit cycle, top, and $(0.91 ; 0.35)$ outside the limit cycle, bottom.

\section{Conclusion}

New models of interacting populations in which one of the two populations exhibits a kind of social behavior have been presented. This socialization is expressed by the fact that they live in a herd, and the influence from the second population is felt essentially by the individuals living at the outskirts of the patch. Mathematically, this situation is modeled via nonlinear Gompertz-like terms. Four basic demographic interactions have been considered, corresponding to symbiotic populations, to obligated mutualism, to the competing case and to predator-prey interactions. In spite of the simplicity of the model, indeed it is just a system of two ordinary differential equations, novel unexpected features are shown to arise. 
For the symbiotic model, results similar to the classical system hold, with only changes in the coexistence equilibrium level.

For the competing case instead, in addition to possibly new different levels for the coexistence equilibrium, which however remains almost always unstable, we observe that the boundary equilibria are unaffected, they are the same as the classical ones. However, there is one case where the coexistence equilibrium exhibits a novel behavior. It occurs for $m>1$ and when (15) is satisfied. In such case it becomes a double equilibrium. The one on the right is stable, the one on the left unstable, Figure 8. Thus, if the initial condition lies to the left of the separatrix, the system's trajectories tend to $P_{3}^{P}$, where only the population $f$ survives. But if the initial condition is on the right of the separatrix, the stable coexistence equilibrium is reached, where both populations survive. Thus the herd behavior, which supposedly constitutes a better defense for the social population, in fact rather saves the individualistic one, avoiding its disappearance. This represents a novel, counterintuitive result.

Finally, contrary to what happens in the classical predator prey model, which has either neutral type of oscillations, when the Lotka-Volterra model is considered, $[1,2,3]$, or which possesses only globally stable equilibria, $[20,22]$, for the case of quadratic mass-action type interactions, we discovered that under suitable conditions on the parameters limit cycles naturally arise in this case. The whole behavior of the predator-prey system is characterized by one single key parameter, $\rho,(12)$. This parameter captures the entire dynamics of the system, expressing the various situations that the model allows. Note that a large $\rho$ is obtained for either high values of the predators' mortality $m$ or a low predators' hunting reward $e$. In fact, for $\rho>1$ the predators population becomes extinct, for $1>\rho>(\sqrt{3})^{-1}$ the predators and prey coexist at stable levels, while at $\rho=(\sqrt{3})^{-1}$ a bifurcation occurs, with the onset of limit cycles. From this value onwards the latter show increasing amplitudes. Thus in the proposed model the coexistence of predators and prey can also be ensured via stable sustained oscillations, triggered by suitable values of $\rho$, see Table 1 .

It is interesting to note that in this last case the model's behavior resembles that of the Holling-Tanner model,

but the latter hinges on completely different assumptions. Thus for the predator-prey case, Michaelis-Menten type terms or the predators' carrying capacity proportional to the prey amount are not necessary to trigger stable sustained oscillations. If the prey gather together in herds and adopt a group defense strategy, coexistence through stable limit cycles arises quite naturally.

Finally, although unable to explicitly construct a Lyapunov function, we have also shown the global stability for the coexistence equilibria of the 
symbiotic model, and for two cases of the predator-prey model, $\rho>1$ and $(\sqrt{3})^{-1}<\rho<1$. This is an additional result worthy to be remarked.

Acknowledgments. The authors thank the referee for the valuable suggestions that contributed to a significant improvement of the paper.

\section{References}

[1] V. Volterra, U. D'Ancona, La concorrenza vitale tra le specie nell'ambiente marino, VIIe Congr. int. acqui. et de pêche, Paris (1931) $1-14$.

[2] A. J. Lotka, Elements of Mathematical Biology, Dover, New York, 1956.

[3] H. Malchow, S. Petrovskii, E. Venturino, Spatiotemporal patterns in Ecology and Epidemiology, CRC, Boca Raton, 2008.

[4] J. D. Murray, Mathematical Biology, Springer Verlag, New York, 1989.

[5] P. H. Leslie, J. C. Gower, The properties of a stochastic model for the predator-prey type of interaction between two species, Biometrika 47 (1960) 219-234.

[6] C. S. Holling, The functional response of invertebrate predators to prey density, Memories Entomol, Soc. Can. 45 (1965) 3-60.

[7] J. T. Tanner, The stability and intrinsic growth rates of prey and predator populations, Ecology 56 (1975) 855-867.

[8] P. A. Braza, The bifurcations structure for the Holling Tanner model for predator-prey interections using two-timing, SIAM. J. Appl. Math. 63 (2003) 889-904.

[9] S. B. Hsu, T. W. Hwang, Y. Kuang, Global analysis of the MichaelisMenten-type ratio-dependent predator-prey system, J. Math. Biol. 42 (2001) 490-506.

[10] S. B. Hsu, T. W., Hwang, Y. Kuang, A ratio-dependent food chain model and its application to biological control, Math. Biosci. 181 (2003) $55-83$.

[11] P. A. Abrams, The fallacies of ratio-dependent predation, Ecology 75(6) (2003) 1842-1850. 
[12] P. A. Abrams, L. R. Ginzburg, The nature of predation: prey dependent, ratio dependent or neither?, Trends. Ecol. Evol. 15 (2000) 337-341.

[13] H. R. Akcakaya, Population cycles of mammals: evidence for ratiodependent predator-prey hypothesis, Ecol. Monogr. 62 (1992) 119-142.

[14] T. Matsuoka, H. Seno, Possibly Longest Food Chain: Analysis of a Mathematical Model, Math. Model. Nat. Phenom. 3(4) (2008) 131-160.

[15] H. I. Freedman, G. Wolkowitz, Predator-prey systems with group defence: the paradox of enrichment revisited, Bull. Math. Biol. 48 (1986) 493-508.

[16] J. Chattopadhyay, S. Chatterjee, E. Venturino, Patchy agglomeration as a transition from monospecies to recurrent plankton blooms, Journal of Theoretical Biology, 253 (2008) 289-295.

[17] V. Ajraldi, E. Venturino, Mimicking spatial effects in predator-prey models with group defense, in J. Vigo Aguiar, P. Alonso, S. Oharu, E. Venturino, B. Wade (Eds), Proceedings of the International Conference CMMSE 2009, 1 (2009) 57-66.

[18] N. Apreutesei, A. Ducrot, V. Volpert, Competition of Species with IntraSpecific Competition, Math. Model. Nat. Phenom. 3(4) (2008) 1-27.

[19] P. Waltman, Competition models in population biology, SIAM, Philadelphia, 1983.

[20] M. Hirsch, S. Smale, Differential equations, dynamical systems, and linear algebra, Academic Press New York, 1974.

[21] A. S. Ackleh, P. Zhang, Competitive Exclusion in a Discrete StageStructured Two Species Model, Math. Model. Nat. Phenom., 4(6) (2009) 156-175.

[22] B. S. Goh, Stability in models of mutualism, The American Naturalist 113 (1979) 261-275. 